\title{
La mer Baltique, un détour par la métaphore du lac
}

\author{
Nicolas ESCACH \\ Maître de conférences \\ Directeur du Campus de Caen \\ Sciences po Rennes (FR) \\ nicolas.escach@sciencespo-rennes.fr
}

\begin{abstract}
Résumé
La mer Baltique semble constamment tiraillée entre son rôle d'appendice maritime ou continental et son statut de laboratoire de la gouvernance transnationale. Constitue-t-elle une simple baie atlantique, une porte au croisement des routes ou au contraire une mer intérieure, à l'écart, quasiment fermée ? La métaphore du lac s'est répandue au début de la décennie 1990 afin d'incarner un vaste processus de régionalisation, facilitant le recours à un imaginaire concret mais mobilisateur. La référence dotait symboliquement la région baltique de l'unité, de l'homogénéité et de la consistance qui lui faisaient défaut. L'intérêt des acteurs politiques et économiques de l'ensemble des niveaux géographiques se portait alors précisément à envisager des connexions renforcées entre les différentes rives. Comment expliquer que le détour par le lac ait été dès lors nécessaire ? Quelles stratégies la substitution du vocable maritime par l'univers lacustre a-t-elle appuyé ?
\end{abstract}

Mots-clés : mer Baltique, lac, territorialisation, marqueur territorial, régionalisation.

\begin{abstract}
The Baltic Sea constantly seems to be torn between its role of maritime or continental appendage and its status of laboratory for transnational governance. Is it merely an Atlantic bay, a door at the crossroads or is it, on the contrary, an isolated and virtually closed inland sea? The metaphor of the lake spread at the beginning of the 1990s to embody a vast process of regionalisation, which facilitated the use of a tangible yet mobilising imagination. This reference symbolically endowed the Baltic Sea Region with the unity, homogeneity and consistency it lacked. At the time, political and economic stakeholders at all geographical levels focused interest precisely on envisaging stronger connections between shores. Why was a detour by the lake therefore necessary? Which strategies has the substitution of maritime terms by the lake universe supported?
\end{abstract}

Keywords: Baltic Sea area, lake, territorialisation, territorial markers, regionalisation. 
Introduction

1. Les fondements géographiques du lac baltique

2. L’instrumentalisation du lac comme support de la régionalisation baltique

3. Du lac à la gestion des biens communs 


\section{La mer Baltique, un détour par la métaphore du lac}

\section{Introduction}

La mer Baltique présente une situation géographique rare en Europe. Divisés par le Rideau de fer au cours de la Guerre froide, les espaces qui en sont riverains sont longtemps restés des glacis se tournant le dos (Mertelsmann and Piirimäe 2012). De nombreuses zones militaires et villes fermées jalonnaient ses rives (quartier de Karosta en Lettonie, villes de Sillamäe et Paldiski en Estonie), créant jusqu'à aujourd'hui des configurations spatiales de no man's land. La chute du Rideau de fer en 1989 et la seconde indépendance des États baltes (1991) s'accompagnent d'un retournement de perspectives et d'un élargissement des imaginaires spatiaux. Une multitude de réseaux d'acteurs de tous les niveaux géographiques émerge, soit ex-nihilo, soit par institutionnalisation de liens informels plus anciens, tissés par-delà les lignes de démarcation européennes. Les territoires baltiques entrent dans une nouvelle bifurcation spatiale, au sens de processus rapide donnant lieu à une transformation du contexte de référence (Thibault 2013). La Guerre froide avait différencié les États plutôt atlantistes (Danemark, Norvège) ou communistes (URSS, Pologne) des États de la «balance nordique » (Suède neutre et Finlande semi-neutre) à la fois poches isolées et points de contact entre les deux blocs (Simoulin 1999). À l’inverse, le début de la décennie 1990 semblait marquer une déconstruction de la frontière est/ouest (Perko 1996 ; Blanc-Noël 2002 ; Kern and Löffelsend 2004), la régionalisation baltique venant combler les blancs de l'ancienne carte.

À mesure du rapprochement des territoires riverains, de nombreuses métaphores sont venues nommer l'unité retrouvée: « Nouvelle Hanse » (en référence aux liens tissés dès les XII ${ }^{\mathrm{e}}$-XIII ${ }^{\mathrm{e}}$ siècles entre différents comptoirs de la Baltique ${ }^{1}$ ), « Méditerranée du Nord », « Mare Balticum » ou " Lac baltique ». Cette dernière expression n'est pas sans rappeler des travaux menés par plusieurs membres de la « nouvelle géographie » à partir de méthodes d'analyse spatiale développées dès les années 1960-1970 par des chercheurs anglophones. Leur objectif est de révéler les structures de l'espace, les lois universelles et les principes

1 Dès le début de la décennie 1980, la Hanse est instrumentalisée pour accélérer la déconstruction de la frontière est/ouest et présenter comme naturel le processus de régionalisation. Un réseau de villes intitulé « Hanse des temps nouveaux » est lancé à Zwolle, précédant nombre d'organisations volontaristes et de projets se référant, par leurs noms ou leurs symboliques, à la Ligue hanséatique (Escach 2014). 
d'organisation qui l'ont fait naître. Ce faisant, ils s'inspirent à plusieurs reprises de la géographie physique pour construire de nouveaux modèles heuristiques (archipel, isthme, pertuis, seuil). En France, Roger Brunet développe ainsi ses " Modèles de méditerranée », parmi lesquels le lac constitue une métaphore de référence (Brunet 1995). Le lac rejoint le rang des concepts applicables à tout type d'espace et sort des particularités écosystémiques avancées par les géographes classiques et les limnologues (chercheurs en sciences des eaux dormantes continentales). Parmi eux, François-Alphonse Forel désignait le lac comme « une masse d'eau stagnante sans communication directe avec la mer, située dans une dépression du sol fermée de tout côté » (Forel 1901, 3).

Traditionnellement, les lacs se distinguent des rivières, fleuves et autres eaux courantes par des eaux qui ne sont pas entraînées dans une direction unique, les courants lacustres, sils existent, étant fantasques et irréguliers. La profondeur, la superficie et le volume sont suffisants pour provoquer un étagement, une zonation et un cubement durable (régionalisation limnique) de son fonctionnement, ce qui différencie les lacs des étangs et des mares (Touchart, Bartout et Nedjai 2014). Les lacs sont enfin des eaux intérieures qui ne communiquent pas avec l'océan et ne sont pas dépendantes de lui. Le continent et non la mer détermine les apports dans les lacs. La grande conséquence de l'absence de communication avec l'océan est le développement d'une identité propre à chaque lac et un fonctionnement en circuit fermé. Si ce dernier est suffisamment ancien, sa manifestation la plus éminente est l'endémisme biologique (Touchart 2000). Sur un plan géophysique, la frontière entre mers et lacs reste très ténue comme en témoigne des toponymies (lac de Tibériade ou mer de Galilée, lac Asphaltite ou mer Morte) ou des classifications (mer Morte, mer Caspienne, mer d'Aral) ambigües. L'appellation de mer peut alors provenir de la dimension, du taux de salinité ou d'un usage vernaculaire ancien. Pour compliquer le tout, une mer peut être un ancien lac. "Curieusement, il n'est pas facile de donner la définition exacte des lacs » rappelait d'ailleurs Brian Timms (Timms 1992, 1).

Le lac des limnologues a profondément nourri la métaphore du lac tissée entre autres par les représentants de la nouvelle géographie. Tout d'abord, bien que le lac ne soit plus considéré aujourd'hui comme un microcosme complet ${ }^{2}$, ce qui rompt avec l'ancienne tradition forelienne, il apparaît avant tout comme un espace borné, refermé, replié, autour duquel les territoires riverains se définissent, interagissent et s'organisent. Le lac est un principe unificateur et circulatoire. De par sa taille,

2 Le lac est désormais intégré dans un véritable « limnosystème » considérant le rôle du bassin d'alimentation (amont), de l'émissaire (aval) et l'influence de nombreuses variables externes physiques (vent, température) et humaines (appropriations et usages variés). 
ni trop petite (le franchissement est nécessaire), ni trop grande (la maîtrise est possible), le lac permet une territorialisation aboutie sur un modèle quasi-terrestre là où la banalisation de l'espace maritime semble inachevée. Les rives, à distance humaine, remplacent un rivage qui longtemps parut indomptable.

En Baltique, l’image du lac a pu accompagner la construction d'un bornage territorial autour de la mer éponyme. Nous entendons ici par «marqueur territorial » une entité spatiale matérielle ou immatérielle, réelle ou fantasmée, qu'un ensemble d'acteurs et/ou un groupe social choisit de mettre en valeur, d'aménager, de reprendre dans ses discours et pratiques spatiales, conférant une image au territoire qu'il habite (effet vitrine et effet miroir) et le différenciant ainsi plus nettement des autres. Grâce à ces marqueurs, le territoire « dessine un champ symbolique semé d'objets patrimoniaux, de hauts lieux emblématiques, investis par la mémoire collective » (Garnier 2004, 28).

La question de savoir si la Baltique est un lac ou non nous apparaît finalement secondaire par rapport à celle consistant à se demander pourquoi la métaphore du lac est apparue et quels acteurs, intérêts, projections elle a pu servir. Il existe bien, comme nous le verrons, un lac physique hérité et une métaphore du lac instrumentalisée, les deux pouvant entrer par moment en contradiction.

\section{Les fondements géographiques du lac baltique}

La métaphore du lac, apparue principalement au début de la décennie 1990, s'appuie en Baltique sur un terreau propice du point de vue géographique. Le lac y prend les allures d'un espace hérité, d'un espace clos, d'un espace approprié et d'un espace verrouillé, selon que l'on raisonne sur un plan géophysique, culturel ou géopolitique.

Sur un plan physique, la mer Baltique a alternativement été un lac et une mer suivant quatre étapes qui ont conduit à sa formation :

a) La fin du Pléistocène (plus ancienne époque du Quaternaire), jusqu’à 12000 ans avant notre ère, constitue une période de refroidissement global au cours de laquelle les glaciers continentaux sont particulièrement étendus. Un vaste inlandsis d'une épaisseur de 2,75 kilomètres recouvre la Fennoscandie et s'étend jusqu'en GrandeBretagne. La surcharge glaciaire engendre une érosion de la cuvette du domaine baltique. Lorsque la calotte scandinave s'amincit sous l'effet de l'interglaciaire du Pléistocène récent, les eaux de fonte s'accumulent d'abord en un lac proglaciaire.

b) Le niveau de la mer augmentant, celle-ci envahit progressivement les estuaires des fleuves et les chenaux proglaciaires rejoignant les bassins de surcreusement. La mer de Yoldia fait alors son apparition 10000 ans avant notre ère, au moment où commence la période de l'Holocène. 
c) Entre 10000 ans et 9000 ans avant notre ère, la croûte terrestre, notamment au niveau de la Suède centrale, connaît un mouvement de rebond isostatique, soit un soulèvement des masses terrestres consécutif à la déglaciation. Le socle continental remonte, particulièrement au sud où se trouvent les détroits reliant la mer de Yoldia à l'océan mondial, ce qui forme un lac nommé lac à Ancylus.

d) 7000 ans avant notre ère, une nouvelle élévation du niveau de la mer et le ralentissement $\mathrm{du}$ mouvement isostatique engendrent une transgression flandrienne formant la mer de Littorines, l'ancêtre de la mer Baltique.

La mer Baltique apparaît ainsi graphiquement comme une mer quasiment fermée, la clôture de l'espace reflétant la genèse des formes. Laurent Touchart définit le lac comme " un plan d'eau continental séparé de la mer » (Touchart 2000). Le dictionnaire Larousse parle quant à lui d'une « nappe d'eau stagnante » et d'une " étendue de liquide qui ne s'écoule pas ». Ces propriétés pourraient presque être appliquées à la lettre à la mer Baltique, d'une superficie de $385000 \mathrm{~km}^{2}$, pour laquelle Nathalie Blanc-Noël évoque un "cadre géographique contraignant » (Blanc-Noël 2002). Les détroits danois (Skagerrak, Kattegat, Petit Belt, Grand Belt et Øresund) forment un verrou dans le passage vers la mer du Nord. La faible profondeur (en moyenne 54 mètres sur l'ensemble de la Baltique, 23 mètres dans le Kattegatt et moins de 10 mètres au niveau de l'Öresund) contraint le passage de supertankers, de grands minéraliers ou des plus imposants porte-conteneurs (Bayou 2014 ; Escach et Serry 2014). Les routes maritimes baltiques ne peuvent être empruntées que par des navires d'un tirant d'eau inférieur à 17,5 mètres. Cela explique la spécificité baltique, avec une importance des rouliers et des lignes courtes. La relative fermeture de la mer engendre également un faible renouvellement des eaux (tous les 25 ans), ce qui a des incidences écologiques majeures. Le taux de salinité, particulièrement bas, décline d'est en ouest : 10 à $13 \%$ en Baltique propre, 5 à $9 \%$ dans le golfe de Finlande et 3 à $7 \%$ dans le golfe de Botnie. L'englacement participe enfin à une accessibilité inégale des territoires riverains. Depuis 1880, la mer a été totalement prise par des glaces compactes à cinq reprises (Lépy 2009). La probabilité d'occurrence annuelle de la glace est de $100 \%$ dans la Baie de Botnie et dans le fond du Golfe de Finlande, de 50 \% dans le nord de la Baltique Propre et de 10 \% dans le Sud de la Baltique.

Au-delà de la seule dimension physique, le motif du lac s'inscrit dans une tradition culturelle ancienne lisible dans le terme "Baltique ». Adam de Brême pourrait avoir utilisé le terme Belt, qui en langue teutonique peut être traduit par « irruption des eaux ». Cette thèse est accréditée par Grotius dans ses écrits ${ }^{3}$.

3 Voir à ce sujet Ménage, Gilles. 1750. Dictionnaire étymologique de la langue françoise. Paris : Briasson, p. 138. Disponible en ligne 
Le mot proviendrait également du latin Balteus, en scandinave Baelt, qui signifie « ceinture ». Il s'agissait de décrire métaphoriquement la ceinture d'eau séparant la presqu'île danoise d'une péninsule scandinave alors souvent considérée comme insulaire. Les principales sagas nordiques évoquent de leurs côtés un lac oriental [Austmarr] notamment la Saga des rois de Norvège de Snorri Sturluson. Dans les différents États riverains qui la bordent, les mots pour désigner la mer Baltique reflètent une diversité de situations : Östersjö en Suédois (et non hav), soit littéralement « lac de l'Est », mais « mer de l'Est » [Itämeri] pour les Finlandais et « mer de l'Ouest » [Läänemeri] pour les Estoniens. Les Allemands nomment la mer Baltique Ostsee, laissant ainsi une ambiguïté entre l’image d'une « mer intérieure » [Binnenmeer] ou d'un lac allemand (Chillaud 2007).

La figure lacustre peut également surgir de manière plus indirecte dans la littérature lorsque celle-ci choisit la mer comme cadre. Marc Auchet a étudié le motif del'eau dans trois textes de référence : une ballade médiévale danoise : Agnès et le Triton, une pièce d'Andersen : La Petite Sirène, et une pièce d'Ibsen : La dame de la mer (Auchet 1997). Dans ces trois textes, le détour par la mer est associé à la nécessité de dépasser le stade de l'enfance, un mode d'existence régressif et narcissique, pour gagner sur terre la maturité de l'âge adulte. La mer présente un profil ambivalent : elle fascine et inquiète en ce qu'elle concentre les zones d'ombre de la vie psychique tout en représentant une composante indispensable du parcours initiatique achevé après le retour sur terre. Les Danois ont de fait deux termes, forgés dès l'époque Viking, désignant des mouvements contradictoires mais complémentaires : «l'appel du large », soit l'envie de s'élancer en mer (udve ou udlængsel) et « la nostalgie » marquant le retour chez soi, l'aspiration à revenir là où sont ses racines (hjemve). La mer n'ouvre pas ici sur le désir mystérieux du rivage vanté par Alain Corbin (Corbin 1988) mais sur une longue parenthèse. Le mouvement géographique de la quête de soi par le voyage en mer n'est pas sans rappeler le parcours clos et circulaire qu'imprime la forme du lac.

Le lac soumet, par sa physionomie, à la tentation de la fermeture. L'histoire de la mer Baltique a conduit plusieurs auteurs à convoquer l'image du lac dans leurs analyses géopolitiques. De taille médiane, le lac, contrairement à la mer, permet la conquête, le contrôle, la domination, l'hégémonie. Là où l’immensité de locéan ne peut jamais vraiment être embrassé, le lac traduit la puissance territoriale dans toute son expression. La maîtrise de l'espace est complète tant sur un plan économique que politique. Le verrouillage du territoire est physique mais aussi politique, social et culturel : le lac affirme l'homogénéité et l'uniformité de son contenu. L'unité politique est tenue et organisée. La Baltique, qui a connu des phases d'unification sous l'influence de puissances riveraines, répond à cette 
problématique comme en témoigne la variété des publications qui l'ont suggéré : « lac hanséatique », « lac suédois » (Tétart 2007 ; Meyer 2013 ; O’Connor 2015), « lac allemand » (Masson 1999 ; Chillaud 2007 ; Mongrenier 2013), « lac russe » (Colson 1841) et enfin «lac européen » (Ortolland et Pirat 2008; Joenniemi 2009). Pour le géopoliticien Matthieu Chillaud, la représentation ouverte (« mer ») ou fermée ( « lac ») de la mer Baltique dans les cultures nationales « n'est pas sans conséquence sur la perception stratégique qu'ils en ont » (Chillaud 2007, 113). Ce dernier se demande avec Gunnar Åselius : « s’il faut considérer la Baltique comme une mer intérieure nord-européenne bien délimitée ou comme un golfe de l'Atlantique est », et il rappelle que « les puissances dominantes dans la région ont pour leur part toujours soutenu que la Baltique [était] une mer fermée (mare clausum) à laquelle les puissances extérieures [ne devaient] pas avoir accès » (Åselius 2001, 83).

\section{L'instrumentalisation du lac comme support de la régionalisation baltique}

Le lac comme métaphore a fait l'objet d'une formalisation géographique de la part de Roger Brunet qui l'a inscrit dans ses « modèles de méditerranée ». Au milieu des années 1990, le terme « méditerranée » est devenu un concept géographique en lui-même, participant d'un débat épistémologique relativement nourri. Celuici est introduit dans la revue L'Espace géographique, au sein d'un numéro spécial auquel contribuent Roger Brunet, Olivier Dollfus et Pierre Gentelle entre autres (Brunet 1995 ; Dollfus 1995 ; Gentelle 1995). Roger Brunet définit la méditerranée comme un ensemble quasi fermé, constitué par un rivage continu, entourant une masse d'eau suffisante. Les liaisons intra-maritime y sont denses : la navigation demeure aisée et les trajets d'assez courte durée (Brunet 1995). Les rives d'une méditerranée ne sont ni trop éloignées, ni trop proches (Brunet et Vanduick 1995). L'auteur présente sept grands modèles de méditerranées : le lac, le foyer, le détroit, l’isthme, le croissant, la barrière, le chott. Dans le cadre d'un lac, la mer est le centre et le principe de liaison. L'accent est mis sur l'interconnexion entre les rives, l'introversion, un fonctionnement systémique en vase clos et la fermeture. L'espace est relativement soudé avec de fortes possibilités de synergies (Brunet 1995). Le fait que le lac tourne le dos à son environnement n'empêche pas des interactions sur les marges extérieures, qu'elles soient menacées, convoitées ou qu'elles permettent des apports externes.

Ces caractéristiques du lac recouvrent partiellement les critères de définition des régions, telles qu'elles sont étudiées par les géographes. Une région est avant tout un espace borné qu'il est possible de distinguer des espaces environnants par 
son unité interne (Goodall 1987). Jacqueline Beaujeu-Garnier la définit d'ailleurs comme « un morceau d'espace qui se distingue de l'espace voisin » avant d'ajouter « mais comment s'en distingue-t-il ? Là commencent les divergences... » (BeaujeuGarnier 1971, 99). En Baltique, une convergence entre scientifiques et acteurs institutionnels est née pour déconstruire la frontière est/ouest en façonnant une unité régionale à coup de formules performatives. Les premiers acteurs établissant des contacts le long des territoires riverains développent dès le début des années 1990 un discours protecteur (favorisant un échange que les dominations, les conflits, les divisions rendaient improbable), prophétique (mobilisant la société civile en nommant l'entité qu'il faudra construire), voire programmatique (annonçant de futurs projets pour un espace fantasmé). Les séminaires et conférences sur l'apparition d'un nouvel espace de coopération en Baltique se multiplient à la même époque ${ }^{4}$ (Helmryd 1993). Le lac répond donc à une exigence de narration. Il offre une image pragmatique pour ceux qui souhaitent accélérer la réunification des deux rives : à l'est pour retrouver "l'Occident kidnappé " (Kundera 1983) ; à l'ouest pour appuyer un mélange de messianisme et d’intérêts bien compris. Il permet de filer la métaphore historique, ouvrant sur un passé dont le rôle reste considérable en Europe centrale et orientale. Comme le rappelle le poète lituanien Tomas Venclova : « là-bas, le passé est vivant, actuel, s'insinue quotidiennement dans les journaux, influe sur le comportement des gens ».

La région de la Baltique est le produit d'une unité factice trop largement fantasmée à l'aide d'images créées ou recréées. Elle est en réalité caractérisée par une diversité de langues, de religions, de systèmes politiques et d'héritages. Au cours de son histoire, elle a été le théâtre de bien plus de conflits que de coopérations. À son propos, il serait plus juste de parler d'une histoire croisée que d'une histoire commune. Les similitudes les plus visibles s'expliquent par un référentiel partagé constitué de symboles, de récits, d'images et de discours qui peuvent directement être mobilisés et sont mobilisateurs. Aujourd'hui encore, les stigmates de la coupure restent bien visibles : un dynamisme des coopérations intra-nordiques et des liens réactivés entre les États baltes et l'est de l'Europe malgré une forte européanisation, une persistance spatiale des territoires et une interconnexion encore partielle (Serry 2005). Même les échanges maritimes, hérités des anciens réseaux hanséatiques et considérés comme les principaux vecteurs d'unité, ne répondent que partiellement au principe d'une couture transnationale. En 2013, sur 114 lignes roulières, un peu moins d'un tiers (30 \%) étaient transnationales (lien entre deux zones), un quart (26\%) avaient pour

4 Séminaire «Zukunftsregion Ostsee » à Travemünde en 1990, colloque « The new Hansa » à Kotka en 1990 et colloque « The Baltic Sea Area: A region in the making » à Tallinn en 1991. 
destination des ports extra-baltiques, alors que $44 \%$ des lignes reliaient deux ports d'une même zone transfrontalière.

L'espace géographique flou de Christiane Rolland-May, un espace aux limites imprécises, plus ou moins nettes ou continues, conviendrait bien mieux que le concept de région pour caractériser l'ensemble baltique. Selon elle, les franges d'un espace géographique flou répondent à une structuration par gradient d'appartenance. Ses composantes, recouvrables, peuvent appartenir à plusieurs espaces à la fois sans être forcément disjointes (Rolland-May 1984 et 1987).

\section{Du lac à la gestion des biens communs}

Que reste-t-il, passé l'effet d'annonce, de l'espace du lac ? Les territoires riverains baltiques, placés en face-à-face autour d'une mer ${ }^{5}$ à l'origine de multiples défis communs, ont constitué depuis la chute du Rideau de fer un modèle de coopération transnationale aux yeux de l'Union européenne. Signée dès 1974 et révisée en 1992, la Convention pour la protection du milieu marin de la mer Baltique, aussi appelée Convention d'Helsinki, est le premier texte-cadre d'envergure rédigé par les États riverains de la mer Baltique (URSS, Pologne, RDA, Danemark, Suède, Finlande) ${ }^{6}$. Suivent la création de l'Union des villes de la Baltique en 1991, du Conseil des États de la mer Baltique en 1992, la mise en place d'une Dimension septentrionale en 1997, suivie d'une stratégie européenne en mer Baltique ${ }^{7}$ censée la relancer en 2009.

Loin de constituer l'apogée d'un rapprochement attendu, la gestion commune du milieu marin, bien quefondamentale, a plutôt contribuéen Baltiqueàl'émergence d'un consensus mou, alors que des questions sécuritaires et géopolitiques majeures restaient non résolues. Des normes plus ou moins respectées (émanant d'ailleurs davantage de l'Organisation maritime internationale (OMI) que de cadres régionaux comme la Convention d'Helsinki) censées prévenir les risques, lutter

5 Des acteurs norvégiens ou biélorusses sont régulièrement associés aux projets baltiques sans être directement riverains. D’autre part, la Russie ne présente qu'une fenêtre étroite sur la mer Baltique à l'extrémité du golfe de Finlande, si l'on excepte l'enclave/exclave de Kaliningrad qui constitue un avant-poste hautement stratégique.

6 La convention délimite la mer Baltique (article 1), puis les pollutions concernées (article 2). Elle aborde l'introduction de substances dangereuses en mer (article 5), la pollution d'origine tellurique (article 6), les rejets volontaires et accidentels des navires, notamment le pétrole, et le déballastage en mer (article 7), l'immersion de déchets (article 9), l'exploration et l'exploitation du sous-sol (article 11), la coopération scientifique et technologique (article 16).

7 La macro-région baltique crée une convergence de réseaux et projets existants orientant les parcours interterritoriaux et la recomposition des niveaux en ciblant stratégiquement les défis pour lesquels la continuité territoriale s'avère la plus nécessaire. Elle dessine une géographie intermédiaire évolutive, principalement intracommunautaire, à géométrie variable et sans assise territoriale et institutionnelle, bien qu'elle soit à l'origine d'entre-deux territoriaux à d'autres échelles d'espace (régions urbaines, coopérations transfrontalières, plateformes transnationales). Elle combine des visions fonctionnelles et transversales avec un leadership administratif pourtant principalement ministériel et étatique (Escach 2020). 
contre la pollution ou réguler le trafic maritime ont relégué au second plan des débats jugés trop polémogènes. Le Conseil des États de la mer Baltique, associant la Russie aux États baltes et aux autres pays riverains, a rapidement écarté les questions de sécurités « dures » au profit d'une coopération sur des sujets moins polémiques comme l'environnement, la culture ou l'économie (Marin 2009). La Baltique n'aurait-t-elle donc rien d'autre à partager que les conséquences de la « tragédie des biens communs ${ }^{8}$ ?

Le lac est devenu un espace de tensions (Bayou 2017 ; Chillaud 2019 ; Escach 2021). Il semblait bien divisé lors des conflits précédant l'arrivée du gazoduc Nord Stream opposant à propos de son tracé la Russie, des pays de transit comme la Pologne ou les États baltes et des pays nordiques questionnant les impacts environnementaux ou une possible instrumentalisation géopolitique9 L'incitation de l'Union européenne à planifier l'espace maritime a également réveillé localement de nombreux conflits d'usage. En Baltique, comme ailleurs, les espaces maritimes ont fait l'objet depuis les années 1970 d'une appropriation croissante dans les domaines aquacoles, industriels ou récréatifs. La dynamique est telle qu'une directive européenne adoptée en 2014 exhorte tous les États membres de l'UE à lancer un processus de planification de leur espace maritime dès septembre 2016 pour un aboutissement à l'horizon 2021. Il s'agit de mettre de l'ordre, par le zonage, dans un développement spatial devenu anarchique puisqu'assuré par une diversité d'acteurs aux intérêts divergents et issus de plusieurs États ou régions. Comme dans le cadre de planification terrestre, l'organisation de l'espace maritime n'est pas exempte d'égoïsme territorial. Les prospectives sur la Baie de Poméranie ont par exemple montré un décalage entre les différents pays. Le Land du Mecklembourg-Poméranie-Occidentale, très attractif pour les touristes, a déjà réalisé un plan régional pour la zone des 12 milles nautiques prévoyant des règles particulièrement drastiques limitant l'implantation d'éoliennes offshore dans son secteur. Cela obligerait les autres pays à accepter, dans le cadre d'une planification conjointe, plusieurs champs d'éoliennes en dehors des eaux territoriales allemandes (Escach 2016).

8 Expression tirée d'un article controversé de l'écologue américain Garrett James Hardin publié dans la revue Science en 1968.

9 Le gazoduc était notamment accusé par les autorités suédoises de constituer un avant-poste d'espionnage, l'installation d'une plateforme de maintenance près de l'île de Gotland cristallisant en particulier les tensions. 


\section{Conclusion}

Le lac baltique est-il voué à s'assécher prenant les allures du chott soumis à l'évaporation? Les États nordiques, marqués par une forte cohésion interne, ont multiplié les contacts avec des partenaires issus de toutes les régions européennes. De leur côté, les États baltes s'imposent de plus en plus comme des relais entre Europe et Asie, érigeant la Baltique en un estuaire pour les voies eurasiatiques. Définir le lac sur un plan métaphorique, comme un système territorial d'échelle intermédiaire pour lequel l'unification interne (topologique puis topographique) entre rives serait plus constitutive et profitable (y compris sur le plan du rayonnement) que des liens tissés directement avec les espaces extérieurs, ne permet pas une application satisfaisante au contexte baltique qui hybride sans cesse les sept modèles de méditerranées proposés par Roget Brunet.

D'autre part, l'image séduisante du lac ne doit pas empêcher de questionner le lien de causalité entre forme géographique, organisation politique et systèmes spatiaux. La Caspienne est aussi un lac potentiel (ou une mer fermée, selon les points de vue) dont les riverains coopèrent mal. À l'inverse, à toutes les échelles, la Baltique offre l'un des plus denses réseaux de coopération du monde, du niveau des villes à celui des États.

L'étude de la région baltique ne réhabilite la métaphore du lac que si elle prend une tournure anatomique, la rapprochant ainsi des ensembles flous décrits par Christiane Rolland-May. Le lac peut ainsi être défini en médecine comme un " espace intertissulaire clos » contenant notamment du sang (lacs sanguins) ou de la lymphe (lacs lymphatiques). Le lac baltique articule à la fois un modèle gravitaire exploitant les vertus de la proximité et une intermédiarité favorisant l'interconnexion ouverte, le recouvrement, l'interpénétration. Le lac forme ainsi une discontinuité de basse intensité marquant plus ou moins l'espace nordeuropéen au gré des reconfigurations temporelles.

\section{Références bibliographiques}

Åselius, Gunnar. 2001. « La géographie militaire suédoise et scandinave dans une perspective historique ». Stratégique 81 : 77-103.

Auchet, Marc. 1997. "Variations sur le thème de la (petite) sirène. Quelques réflexions sur la symbolique de l'eau dans les littératures scandinaves ». In Anthropologie de l'eau, 55-66. Nancy : Presses universitaires de Nancy.

Bayou, Céline. 2014. « Baltique. Une mer en péril ».P@ges Europe. La Documentation française. Bayou, Céline. 2017. " Les États baltes face à la Russie », Politique internationale 155.

Beaujeu-Garnier, Jacqueline. 1971. La Géographie, méthodes et perspectives. Paris : Éditions Masson. 
Blanc-Noël, Nathalie. 2002. La Baltique : une nouvelle région en Europe. Paris : L'Harmattan. Brunet, Roger. 1995. " Modèles de méditerranées ». L’Espace Géographique 24 (3) : 200-202.

Brunet, Roger, et Régine Vanduick. 1995. " Le concept de "Méditerranée" ». L’Espace Géographique 24 (3) : 209-222.

Chillaud, Matthieu. 2007. " La démarche stratégique des États baltes dans l'architecture européenne de sécurité et de défense. Une politique fondée sur une dialectique identitaire et militaire. De la restauration de leur indépendance aux commémorations russes du soixantième anniversaire de la victoire contre l'Allemagne nazie ". Thèse de doctorat, Université Montesquieu-Bordeaux IV.

Chillaud, Matthieu. 2019. « L'espace Baltique entre périls stratégiques et angoisses sécuritaires ». Stratégique 121-122: 15-59.

Colson, Félix. 1841. De la Pologne et des cabinets du Nord, Tome 2. Paris : Paulin.

Corbin, Alain. 1988. Le territoire du vide. L'Occident et le désir du rivage, 1750-1840. Paris : Aubier.

Dollfus, Olivier. 1995. " Méditerranées, essai d’analyse géographique ». Espace géographique 24 (3), 194-199.

Escach, Nicolas. 2014. " Réseaux de villes et recompositions interterritoriales dans l'espace baltique ». Thèse de doctorat, École Normale Supérieure de Lyon.

Escach, Nicolas, et Arnaud Serry. 2014. "Les "méditerranées" ou l'émergence de régionalisations réticulaires ". In Géographie des mers et des océans, éd. Nicolas Escach (dir.), 226-270. Paris : Dunod.

Escach, Nicolas. 2016. " De la mer en partage au partage de la mer ». Monde diplomatique 07/2016.

Escach, Nicolas. 2020. "Baltic Sea macro-region". In Critical Dictionary on Cross Border Cooperation in Europe, ed. Birte Wassenberg and Bernard Reitel. Brussels: PIE - Peter Lang.

Escach, Nicolas. (2021, à paraître). "Vulnérabilités et recompositions baltiques : les conséquences d'une géopolitique insidieuse ». Mappemonde.

Forel, François-Alphonse. 1901. Handbuch der Seenkunde: Allgemeine Limnologie. Stuttgart: J. Engelhorn.

Garnier, Edwige. 2004. « Une contribution àl'approchedu territoire et de la ressource territoriale: le cas du marqueur territorial, notamment pour les populations déterritorialisées ».Montagnes Méditerranéennes 20 : 25-33.

Gentelle, Pierre. 1995. " À propos de méditerranée : de la géo à la néographie ». Espace géographique 24 (3) : 203-208.

Goodall, Brian. 1987. Dictionary of Human Geography. New York: Penguin Books.

Helmryd, Cécile. 1993. « La Baltique : regards nordiques sur une région en devenir ». Mémoire de fin d'études, Université de Paris 1.

Joenniemi, Pertti. 2009. “The EU Strategy for the Baltic Sea Region, a catalyst for what?”. DIIS Brief. Danish institute for international studies.

Kern, Kristine, and Tina Löffelsend. 2004. "Governance beyond the nation-state: Transnationalization and Europeanization of the Baltic Sea Region”. Discussion Papers. 
Research Unit: Civil Society and Transnational Networks, WZB Berlin Social Science Center.

Kundera, Milan. 1983. « Un Occident kidnappé ou la tragédie de l'Europe centrale ». Le débat $27: 3-23$.

Lépy, Élise. 2009. " Les glaces de mer en mer Baltique. Étude géographique et implications environnementales et sociétales à partir de l'étude compare de la Baie de Botnie (Oulu, Finlande) et du Golfe de Riga ». Thèse de doctorat, Université de Caen.

Marin, Anaïs. 2009. « Argument Baltique : faux prétexte et modèle juste ». Outre-Terre 23 : 347362.

Masson, Philippe. 1999. Histoire de l'armée allemande 1939-1945. Paris : Perrin.

Mertelsmann, Olaf, and Kaarel Piirimäe. 2012. The Baltic Sea Region and the Cold War. Francfort-sur-le-Main: Peter Lang International Academic Publishers.

Meyer, Philippe. 2013. Baltiques. Histoire d'une mer d'ambre. Paris : Perrin.

Mongrenier, Jean-Sylvestre. 2013. " La Baltique n'est pas une méditerranée du Nord ». Communication en ligne sur le site de l'Association des Auditeurs de l'Institut des Hautes Études de Défense Nationale. Région Nord-Pas de Calais-Belgique-Luxembourg.

O'Connor, Kevin C. 2015. The History of the Baltic States, $2^{\text {nd }}$ Edition. The Greenwood Histories of the Modern Nations. Santa Barbara, Denver: Greenwood.

Ortolland, Didier, et Jean-Pierre Pirat. 2008. Atlas géopolitique des espaces maritimes, Frontières, énergie, pêche et environnement. Paris : Éditions Technip.

Perko, Susanna. 1996. Nordic-Baltic Region in Transition. Tampere: Tampere Peace Research Institute.

Rolland-May, Christiane. 1984. " Notes sur les espaces géographiques flous ». B.A.G.F. 502 : 159-164.

Rolland-May, Christiane. 1987. " La théorie des ensembles flous et son intérêt en géographie ». L'Espace Géographique 16 (1) : 42-50.

Serry, Arnaud. 2005. " Les ports de la Baltique orientale : persistance des territoires et mutations ". Le courrier des pays de l'Est $1048: 40-50$.

Simoulin, Vincent. 1999. La coopération nordique, l'organisation régionale de l'Europe du Nord depuis la tentative autonome jusqu’à l'adaptation à l'Europe. Paris : L'Harmattan.

Sturluson, Snorri. 2000. Heimskringla, Histoire des rois de Norvège, première partie traduite par François-Xavier Dillmann. Paris : Gallimard.

Tétart, Frank. 2007. Géopolitique de Kaliningrad : une « île » russe au sein de l'Union européenne élargie. Paris : Presses Universitaires de Paris-Sorbonne.

Thibault, S. 2013. « Bifurcation ». In Dictionnaire de la géographie et de l'espace des sociétés. Paris : Belin.

Timms, Brian. 1992. Lake Geomorphology. Adelaide: Gleneagles.

Touchard, Laurent. 2000. "Qu'est-ce qu'un lac ? ». Bulletin de l'Association de Géographes Français 77 (4) : 313-322.

Touchart, Laurent, Pascal Bartout, et Rachid Nedjai. 2014. « La géographie limnologique en France : conjugaison de l'espace et du temps pour la compréhension des relations hommemilieu ». Bulletin de la Société géographique de Liège 62 : 93-103. 